\title{
Cervical restorations useful when assessing gingival and periodontal health
}

SADJ November 2018, Vol. 73 No. 10 p633 - p634

A Volchansky

"Once a Periodontal Patient, always a Periodontal Patient".

This comment could be describing the affliction of Chronic Periodontitis or Refractory Periodontitis which refer to the periodontal status of patients who require monitoring over extended periods and who demonstrate severe attachment loss (derived from Parameters of Care: Journal of Periodontology, 20001).

The progression of periodontal diseases is assessed by the extent of gingival recession, the severity of clinical attachment loss, and the probing depths of pockets. Periodontal disease is generally described as a slow and continually progressive condition. ${ }^{2}$ It may well be important that a fixed reference point is available to ensure repeatability when these measurements are recorded. The landmark habitually used is the cemento-enamel -junction (CEJ). The normal gingival margin position is 0.5 $-2.0 \mathrm{~mm}$ coronal to the CEJ. Gingival recession is defined as the increase in the location of the gingival margin apical to the CEJ. ${ }^{4}$

A cervical restoration is one that is placed adjacent to the CEJ or the gingival margin ( $G \vee$ Black $\left(1902^{3}\right.$ ). The margins of such a restoration are clearly visible adjacent to the gingival attachment and the location may approximate the cervical line / cemento-enamel junction of the tooth.

It may be suggested that a point on the apical or gingival margin of a conveniently placed cervical restoration could be used as a measuring point in relation to the determination of gingival or periodontal health, should the CEJ not be visible or be obscured by a restoration, as seen in Figure 1.

The preferred proximity of the restoration, and/or the margin of a synthetic crown, to the periodontal structures is debatable.

\section{Author affiliations:}

1. Alfred Volchansky, BDS Dunhelm PhD Wits, Sessional Specialist, Department of Oral Medicine and Periodontology, School of Oral Health Science, University of the Witwatersrand, Johannesburg, South Africa.

Corresponding author: Alfred Volchansky

Department of Oral Medicine and Periodontology, School of Oral Health

Science, University of the Witwatersrand, Johannesburg, South Africa.

Email: volchans@iafrica.com

\section{ACRONYM \\ CEJ: Cemento-enamel-junction}

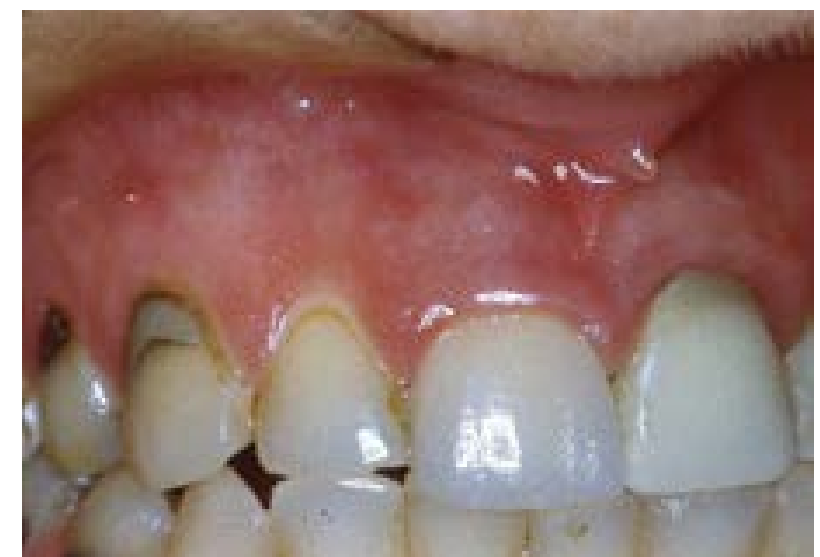

Figure 1. Cervical restorations on $13 ; 14$, obscuring the CEJ.

There are three options, supragingival, equigingival and subgingival. Much has been written about the importance of the restorative margin, its location, the materials and the contours of any restoration in relation to periodontal health. ${ }^{5,6}$

Supra-gingival placement is recommended as the location of choice for all restorative margins to avoid iatrogenic periodontal disease. ${ }^{7,8}$ However, the circumstances determining the placement of a cervical restoration may dictate the final location of the margins. This may be influenced by the reasons for the placing the filling ...for example the extent of decay, for aesthetics or to control sensitivity.

Figure 1 shows a cervical restoration ${ }^{2}$ on the buccal aspect of tooth 13, obscuring the CEJ, while the CEJ and gingival margin are clearly visible on the adjacent crown of tooth 12 .

\section{CLINICAL CASE}

This case demonstrates the use of the apical margin of a cervical restoration as a fixed point for the measurement of gingival recession and as an indicator of the extent of attachment loss. 
Figure 2 is of a patient seen at the initial consultation, where marked gingival inflammation was noted distal to tooth 43. The adjacent tooth has a cervical restoration and any gingival recession for this tooth could be measured from the apical aspect of the restoration to the gingival margin. Periodontal treatment was advocated. However, the patient re-appeared four years later, not having had any periodontal care (Figure 3 ).

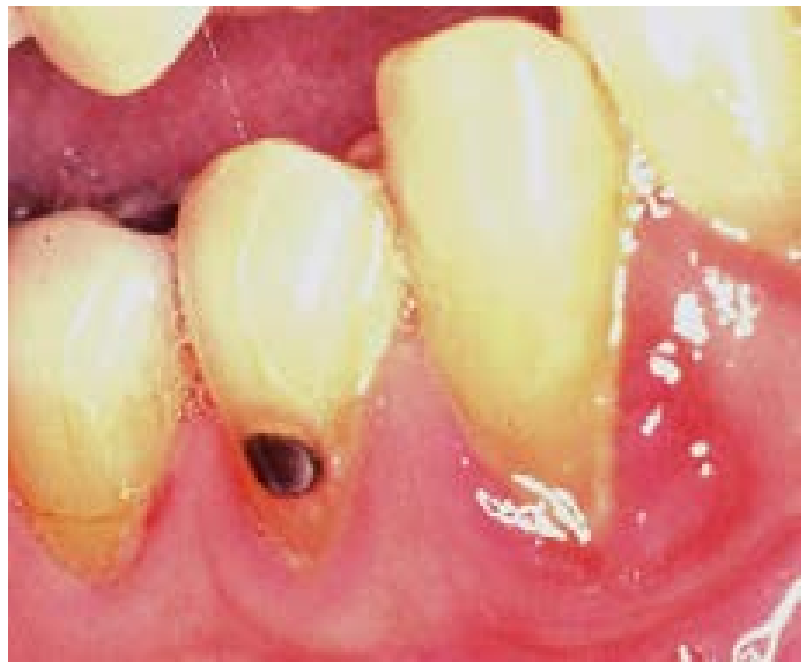

Figure 2. Initial consultation.

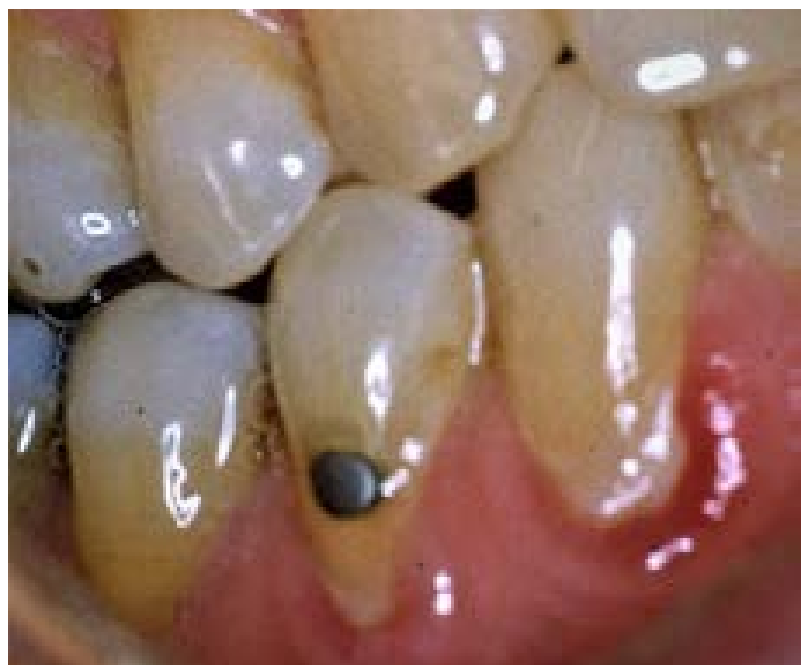

Figure 3. Four years later.
The inflammation distal to tooth 43 appeared to be slightly reduced. Of relevance was the measurement of recession on the adjacent tooth, recorded as recommended using the apical aspect of the restoration as a fixed reference point. Patently an increase had occurred in that dimension.

\section{DISCUSSION}

The case illustrates the use of a landmark provided by a restoration as a fixed point for the measurement of gingival recession and offers an indication of the progress of attachment loss.

\section{CONCLUSION}

Where the CEJ is obscured, the apical margin of a cervical restoration may provide a practical solution to a clinical problem.

\section{REFERENCES}

1. Parameters of Care. Refractory Periodontitis. 2000. Journal of Periodontology: 71: 9SUPP) 859-60.

2. Burt BA. Periodontitis and aging: reviewing evidence. J Am Dental Assoc. 1994;25:273-9.

3. Black GV. Derived from "Class $\vee$ caries, affecting the $1 / 3$ of the facial or lingual surfaces of anterior or posterior teeth". Wikipedia. Org.

4. Glossary of Periodontal Terms 2001. American Academy of Periodontology.

5. Arenberg P. Marginal fit and cervical extent of Class II amalgam restorations related to periodontal conditions. J Periodontology. 1980;17:67-72.

6. Padbury A, Eber R, Hom-Lay Wang.. Interactions between the gingiva and the margin of restorations. J Clin Periodontology. 2003;30:379-85.

7. Brunsvold MA, Lane JJ. 1990. (Overhanging dental restorations). J Clin Periodontology. 17:67-72

8. Reeve G R. 1991. Restorative margin placement and periodontal health. J Pros Dent. 66:733-736. 